\title{
A regularized heat trace for hyperbolic Riemann surfaces of finite volume
}

\author{
Jay Jorgenson and Rolf Lundelius*
}

Abstract. Let $M$ denote a hyperbolic Riemann surface of finite volume, and let $K_{M}(t, x, y)$ be the heat kernel associated to the hyperbolic Laplacian which acts on the space of smooth functions on $M$. If $M$ is compact, then we have the equality

$$
\int_{M} K_{M}(t, x, x) d \mu(x)=\sum_{n=0}^{\infty} e^{-\lambda_{n} t},
$$

where $\left\{\lambda_{n}\right\}$ is the set of eigenvalues of the Laplacian. If $M$ is not compact, then it is well known that the heat kernel exists yet is not of trace class. In this paper we will define a regularized heat trace associated to any hyperbolic Riemann surface of finite volume, compact or noncompact. After we have defined the regularized heat trace, we study the asymptotic behavior of the regularized heat trace on a family of degenerating hyperbolic Riemann surfaces. Our results involve pointwise convergence and uniformity of asymptotic expansions in the pinching parameters. In particular, we study uniformity of long time asymptotics of the regularized heat trace minus the contribution from the small eigenvalues by analyzing the Poisson kernel and Dirichlet heat kernel in a finite cylindrical neighborhood of the pinching geodesics. As applications of our results, we are able to study asymptotic expansions of the Selberg zeta function and spectral zeta function on degenerating families, both improving known results in the compact setting and proving new results in the non-compact situation. Results from this article have been extended to the setting of degenerating hyperbolic three manifolds of finite volume in [DJ1] and [DJ2].

Mathematics Subject Classification (1991). 58G18, 58G11, 58G25.

Keywords. Riemann surfaces, heat kernels, zeta functions.

*The first author acknowledges support from NSF grants DMS-93-07023 and DMS-96-22535, from the Max-Planck-Institut für Mathematik, and from the Sloan Foundation. The current address of the first named author is: Department of Mathematics, Oklahoma State University, Stillwater, OK, 74078. The current address of the second named author is: Salomon Brothers Inc, 7 World Trade Center, New York, NY 10048. 


\section{$\S 0$. Notation and background material}

This section contains a brief discussion of the geometry of degenerating families of hyperbolic Riemann surfaces of finite volume. For further details, the reader is referred to $[\mathrm{A} 1],[\mathrm{A} 2],[\mathrm{B}]$ and $[\mathrm{R}]$. We will follow the notation and conventions established in [JLu1] and [JLu2], which will be assumed throughout this paper.

Let $\mathbf{h}$ be the upper half plane model of the hyperbolic plane, equipped with its standard metric of constant curvature equal to -1 and distance function $d_{\mathbf{h}}$. Throughout this paper, all metrics $d \mu$ will be hyperbolic metrics.

For $q>0$, let $C_{q}$ denote the hyperbolic, infinite cylinder with simple, closed geodesic of length $q$, which can be described explicitly by

$$
C_{q}=\{(\tau, \theta): \tau \in \mathbf{R} \text { and } \theta \in \mathbf{R} / \mathbf{Z}\}
$$

with length form

$$
d s^{2}=d \tau^{2}+q^{2} \cosh ^{2}(\tau) d \theta^{2} .
$$

A convenient fundamental domain for $C_{q}$ in $\mathbf{h}$ is

$$
\{\rho \exp (i \alpha): 1 \leq \rho<\exp (q), \quad 0<\alpha<\pi\}
$$

with hyperbolic metric induced from $\mathbf{h}$ and uniformizing group $\{\exp (k q): k \in \mathbf{Z}\}$ which acts on $\mathbf{h}$ by multiplication. For any $\varepsilon>0$, let $C_{q, \varepsilon}$ denote the submanifold of $C_{q}$ obtained by restricting $|\tau|<\sinh (\varepsilon /(2 q))$. A fundamental domain for $C_{q, \varepsilon}$ in $(0.1)$ is obtained by adding the restriction

$$
\cot ^{-1}(\varepsilon /(2 q))<\alpha<\pi-\cot ^{-1}(\varepsilon /(2 q)) .
$$

An easy calculation shows that the volume of $C_{q, \varepsilon}$ is $\varepsilon$, and the length of each boundary component of $C_{q, \varepsilon}$ is $\left(q^{2}+\varepsilon^{2} / 4\right)^{1 / 2}$. The measure on the boundary of $C_{q, \varepsilon}$ induced from the hyperbolic metric will be denoted by $d \varrho$.

Let $C_{0}$ denote an infinite cusp, which is the non-compact Riemann surface isometric to the punctured unit disc with complete hyperbolic metric. A fundamental domain for $C_{0}$ in $\mathbf{h}$ is

$$
\{x+i y: y>0 \text { and } 0 \leq x<1\}
$$

with group action which identifies the boundary points $i y$ with $1+i y$. For any $\varepsilon>$ 0 , let $C_{0, \varepsilon}$ denote the submanifold of $C_{0}$ obtained by restricting the $y$ coordinate of (0.2) to $y>2 / \varepsilon$. The volume of $C_{0, \varepsilon}$ is $\varepsilon / 2$, and the length of the boundary of $C_{0, \varepsilon}$ is $\varepsilon / 2$. Again, the induced boundary measure will be denoted by $d \varrho$.

Given a $p$-tuple $\ell=\left(\ell_{1}, \ell_{2}, \cdots, \ell_{p}\right)$ of positive real numbers, let $|\ell|$ be the supnorm of $\ell$. Let $C_{\ell}$ be the disconnected surface $C_{\ell}=\cup C_{\ell_{k}}$. Given $\varepsilon>0$, let $C_{\ell, \varepsilon}$ be the disconnected surface $C_{\ell, \varepsilon}=\cup C_{\ell_{k}, \varepsilon}$. The family of surfaces $\left\{C_{\ell}\right\}$ with $|\ell| \rightarrow 0$ is called a degenerating family of hyperbolic cylinders of infinite volume. 
For notational convenience, we will write $\ell \rightarrow 0$ when $|\ell| \rightarrow 0$ and $\ell>0$ to mean all $\ell_{k}>0$.

In [JLu1] and [JLu2] we gave a construction of a degenerating family $M_{\ell}$ of either compact or non-compact hyperbolic Riemann surfaces of finite volume. The construction we follow allows one to define unambiguously various notions such as the tracking of points through degeneration and the fixing of points not contained on pinching geodesics. The reader is referred to these articles for complete details, which will be assumed here. The description of the degeneration of $M_{\ell}$ to the limit surface $M_{0}$ also applies to the degeneration of $C_{\ell}$ and $C_{\ell, \varepsilon}$ to their limit surfaces, $2 p \times C_{0}$ and $2 p \times C_{0, \varepsilon}$, respectively. In particular, one has convergence of the hyperbolic metric uniformly away from from the developing cusps.

Let $M_{\ell}$ be a degenerating family of connected, hyperbolic Riemann surfaces with $p$ pinching geodesics. Although each $M_{\ell}$ is connected when $\ell>0$, the limit surface $M_{0}$ need not be connected. In addition, the number of cusps on $M_{0}$ is equal to the number of cusps on $M_{\ell}$ plus $2 p$. For any $0<\varepsilon<1 / 2$, the surface $C_{\ell, \varepsilon}$ embeds isometrically into $M_{\ell}$ (see [R]). The surface $M_{0}$ contains $2 p$ embedded copies of $C_{0, \varepsilon}$ which is the limit of $C_{\ell, \varepsilon} \subset M_{\ell}$. The family of hyperbolic metrics converges uniformly on $M_{\ell} \backslash C_{\ell, \varepsilon}$ (see [A2]). The heat kernel on $M$ associated to the hyperbolic metric will be written as $K_{M}(t, x, y)$, where $t>0$ and $x, y \in M$. If $M_{\ell}$ is a degenerating family, we will denote the family of heat kernels by $K_{\ell}(t, x, y)$. Similarly, the heat kernel on $C_{\ell}$ will be written as $K_{C_{\ell}}(t, x, y)$. On a non-connected surface $M$, if $x$ and $y$ lie on different components, $K_{M}(t, x, y)$ is defined to be zero for all values of $t$. The reader is referred to [JLu1] and [JLu2] for various properties of hyperbolic heat kernels, which will be assumed throughout this paper.

\section{$\S 1$. Regularized heat traces}

Let $M$ be a connected hyperbolic Riemann surface of finite volume, either compact or non-compact. For now, let us assume that $M$ is connected, so then $M$ can be realized as the quotient manifold $\Gamma \backslash \mathbf{h}$, where $\mathbf{h}$ is the hyperbolic upper half space and $\Gamma$ is a discrete group of isometries of $\mathbf{h}$. Let $K_{\mathbf{h}}(t, \tilde{x}, \tilde{y})$ be the heat kernel on $\mathbf{h}$. We shall assume known that the hyperbolic heat kernel on $\mathbf{h}$ is a function of $t>0$ and of the hyperbolic distance of $\tilde{x}$ to $\tilde{y}$, so

$$
K_{\mathbf{h}}(t, \tilde{x}, \tilde{y})=K_{\mathbf{h}}\left(t, d_{\mathbf{h}}(\tilde{x}, \tilde{y})\right) .
$$

Quoting from page 246 of [Ch], we have, for $\rho>0$

$$
K_{\mathbf{h}}(t, \rho)=\frac{\sqrt{2} e^{-t / 4}}{(4 \pi t)^{3 / 2}} \int_{\rho}^{\infty} \frac{u e^{-u^{2} / 4 t} d u}{[\cosh u-\cosh \rho]^{1 / 2}}
$$

with

$$
K_{\mathbf{h}}(t, 0)=\frac{1}{2 \pi} \int_{0}^{\infty} e^{-\left(1 / 4+r^{2}\right) t} \tanh (\pi r) r d r
$$


The heat kernel $K_{M}(t, x, y)$ on $M$ can be written as a periodization of the heat kernel on the universal cover $\mathbf{h}$, meaning

$$
K_{M}(t, x, y)=\sum_{\gamma \in \Gamma} K_{\mathbf{h}}(t, \tilde{x}, \gamma \tilde{y})
$$

where $\tilde{x}$ and $\tilde{y}$ are any points in $\mathbf{h}$ which are $\Gamma$ lifts of the points $x$ and $y$ in $M$. The convergence of (1.1) will be addressed in the proof of Theorem 1.1(a).

Let $H(\Gamma)$ denote a set of representatives of inconjugate primitive hyperbolic classes in $\Gamma$ (meaning classes with $|\operatorname{Tr}(\gamma)|>2$ for any representative in the class), and let $P(\Gamma)$ denote a set of representatives of inconjugate, non-identity, primitive parabolic classes in $\Gamma$ (meaning classes with $|\operatorname{Tr}(\gamma)|=2$ for any representative in the class). If $M$ is compact, then $P(\Gamma)$ is empty. Let $\Gamma_{\gamma}$ denote the centralizer of $\gamma \in \Gamma$. We can use elementary theory of Fuchsian groups, as in the derivation of the Selberg trace formula, to write (1.1) as

$$
\begin{aligned}
K_{M}(t, x, y)=K_{\mathbf{h}}(t, \tilde{x}, \tilde{y}) & +\sum_{n=1}^{\infty} \sum_{\gamma \in P(\Gamma)} \sum_{\kappa \in \Gamma / \Gamma_{\gamma}} K_{\mathbf{h}}\left(t, \tilde{x}, \kappa^{-1} \gamma^{n} \kappa \tilde{y}\right) \\
& +\sum_{n=1}^{\infty} \sum_{\gamma \in H(\Gamma)} \sum_{\kappa \in \Gamma / \Gamma_{\gamma}} K_{\mathbf{h}}\left(t, \tilde{x}, \kappa^{-1} \gamma^{n} \kappa \tilde{y}\right) .
\end{aligned}
$$

We now have the following theorem, which defines what we call the hyperbolic heat trace associated to $M$.

Theorem 1.1. Let $M$ be a connected, hyperbolic Riemann surface of finite volume with $p$ cusps, and assume notation as above.

a) For each $t>0$, the sum

$$
\operatorname{HK}_{M}(t, x)=\sum_{n=1}^{\infty} \sum_{\gamma \in H(\Gamma)} \sum_{\kappa \in \Gamma / \Gamma_{\gamma}} K_{\mathbf{h}}\left(t, \tilde{x}, \kappa^{-1} \gamma^{n} \kappa \tilde{x}\right)
$$

is a well-defined function of $x \in M$.

b) Let $\langle\gamma\rangle$ be the cyclic group generated by $\gamma \in H(\Gamma)$, and let $C_{\gamma}=\langle\gamma\rangle \backslash \mathbf{h}$ be an infinite cylinder. Then we have the equality

$$
\begin{aligned}
\operatorname{HTr} K_{M}(t) & =\int_{M} \operatorname{HK}_{M}(t, x) d \mu(x) \\
& \left.=\frac{1}{2} \sum_{\gamma \in H(\Gamma)_{C_{\gamma}}} \int_{C_{\gamma}}(t, x, x)-K_{\mathbf{h}}(t, 0)\right] d \mu(x) .
\end{aligned}
$$


c) Let $\cup C_{0}$ be $p$ copies of the punctured unit disk in $\mathbf{C}$ equipped with the complete hyperbolic metric, and identify $\cup C_{j, \varepsilon}$ with the isometric neighborhood of the cusps of $\cup C_{0}$. Given any $0<\varepsilon<1 / 2$ and with $j=1, \ldots, p$, we have

$$
\begin{aligned}
\operatorname{HTr} K_{M}(t) & =\int_{M \backslash \cup C_{j, \varepsilon}}\left[K_{M}(t, x, x)-K_{\mathbf{h}}(t, 0)\right] d \mu(x) \\
& +\int_{\cup C_{j, \varepsilon}}\left[K_{M}(t, x, x)-K_{C_{0}}(t, x, x)\right] d \mu(x) \\
& -\int_{\cup C_{0} \backslash \cup C_{j, \varepsilon}}\left[K_{C_{0}}(t, x, x)-K_{\mathbf{h}}(t, 0)\right] d \mu(x) .
\end{aligned}
$$

d) The function $\operatorname{HTr} K_{M}(t)$ is finite for all $t>0$ and has the asymptotic behavior

$$
\operatorname{HTr} K_{M}(t)=O\left(e^{-c / t}\right) \quad \text { as } t \rightarrow 0 \text {, for some } c>0
$$

and

$$
\operatorname{HTr} K_{M}(t)=O(1) \quad \text { as } t \rightarrow \infty .
$$

Proof. For the part (a), first note that the sum is at least formally well-defined since $H(\Gamma)$ is fixed under conjugation. What remains is to argue that the sum converges. Let $N_{\Gamma}(x ; \rho)$ be the number of geodesic paths on $M$ which connect $x$ to itself and which have length at most $\rho$. An elementary argument involving hyperbolic geometry shows

$$
N_{\Gamma}(x ; \rho)=O\left(e^{\rho}\right)
$$

where the implied constant depends on the injectivity radius at $x$. With this bound, together with the estimate

$$
K_{\mathbf{h}}(t, \rho)=O\left(e^{-c \rho^{2}}\right),
$$

with a constant $c>0$ which depends on $t$, part (a) follows. Observe that the above bounds also imply the convergence of (1.1).

Part (b) follows from formal unfolding of the integral over the fundamental domain, as in the derivation of the trace formula. Note that for any $x \in C_{\gamma}$, we have

$$
\sum_{n=1}^{\infty} K_{\mathbf{h}}\left(t, \gamma^{n} \tilde{x}, \tilde{x}\right)=\frac{1}{2}\left[K_{C_{\gamma}}(t, x, x)-K_{\mathbf{h}}(t, 0)\right],
$$

since the fundamental group of $C_{\gamma}$ is isomorphic to $\mathbf{Z}$. This accounts for the factor of $1 / 2$ in (b). The reader is referred to [He2], [M] or [Se] for further details.

Part (c) also is proved by formal unfolding of the integral, provided one can show that the above integrals are finite for all $t$ and their sum is independent of 
$\varepsilon$. For this, one applies a bound from [JLu3] and applications of the maximum principle as [JLu2]. Details are as follows.

As stated, it suffices to show finiteness for all $t$, for then the fact that the relative heat trace is independent of $\varepsilon$ follows from formal adding and subtracting of the integrals. The first integral is bounded since the integrand bounded over the compact range of integration. Further, observe that from the realization of the heat kernel on $M$ as the periodization of the heat kernel on $\mathbf{h}$, we have the estimate

$$
\int_{M \backslash \cup C_{j, \varepsilon}}\left[K_{M}(t, x, x)-K_{\mathbf{h}}(t, 0)\right] d \mu(x)=O\left(e^{-c / t}\right)
$$

for some $c$ which depends on the injectivity radius of the range of integration.

The finiteness of the second integral follows from the bound

$$
\int_{\cup C_{0} \backslash \cup C_{j, \varepsilon}}\left[K_{M}(t, x, x)-K_{C_{0}}(t, x, x)\right] d \mu(x) \leq \frac{p}{2} \cdot \frac{2 \exp (-t / 4)}{(\pi t)^{1 / 2}}\left(\frac{\varepsilon^{2}}{8}\right)^{-\eta} \zeta_{\mathbf{Q}}(1+2 \eta),
$$

where $\eta=\log \left(1+\varepsilon^{2} / 8\right) / 4 t$. For a proof of (1.2), the reader is referred to Theorem 3.1 of [JLu3].

It now remains to show finiteness of the integral over the cusps. For this, the key observation is to note that as a function of $x$ with fixed $y$ in $\cup C_{j, \varepsilon}$ such that $x$ and $y$ lie in the same cusp, the difference

$$
D(t, x, y)=K_{M}(t, x, y)-K_{C_{0}}(t, x, y)
$$

satisfies, as a function of $x$ and $t$, the heat equation. Fix an $\varepsilon_{0}>\varepsilon$ so that all cusps have hyperbolic neighborhoods of area $\varepsilon_{0}$. By the maximum principle (see [Ch], page 180), the maximum of $D(t, x, y)$ will take place when $x$ is on the boundary of the cusp $C_{j, \varepsilon_{0}}$, keeping in mind that $y$ remains in the interior of the smaller cusp $C_{j, \varepsilon}$. Combining this application of the maximum principle with the positivity of the heat kernels, we obtain the bounds

$$
-\sup _{\substack{z \in \partial C_{j, \varepsilon_{0}} \\ 0 \leq \tau \leq t}} K_{C_{0}}(\tau, z, y) \leq D(t, x, y) \leq \sup _{\substack{z \in \partial C_{j, \varepsilon_{0}} \\ 0 \leq \tau \leq t}} K_{M}(\tau, z, y)
$$

For each $z$, the terms in (1.3) satisfy the heat equation on $C_{j, \varepsilon_{0} / 2}$ with zero initial data. Through a second application of the maximum principle, we obtain the bounds

$$
-\sup _{\substack{z \in \partial C_{j, \varepsilon_{0}} \\ w \in \partial C_{j, \varepsilon_{0} / 2} \\ 0 \leq \tau \leq t}} K_{C_{0}}(\tau, z, w) \leq D(t, x, y) \leq \sup _{\substack{z \in \partial C_{j, \varepsilon_{0}} \\ w \in \partial C_{j, 0_{0} / 2} \\ 0 \leq \tau \leq t}} K_{M}(\tau, z, w)
$$


Standard bounds for the heat kernel (see, for example, page 198 of [Ch]), (1.4) provide upper and lower bounds for $D(x, y, t)$ that are independent of $\varepsilon$. Therefore, the integral over the cusps can be made arbitrarily small since the area of the cusp $C_{j, \varepsilon}$ can be made arbitrarily small, thus showing that the relative heat trace is a well-defined function of $t$. We remark here that the lower bounds in (1.3) and (1.4) can be improved trivially to zero combining (1.1) with the observation that the fundamental group of $C_{0}$ embeds into the fundamental group of $M$.

Finally, part (d) follows from the derived upper bounds (1.2) and (1.4) together with the convergence of the heat kernel on the region $M \backslash \cup C_{j, \varepsilon}$, as given by Theorem 1 of [JLu1] or Theorem 1.3 of [JLu3].

Definition 1.2. Let us define the regularized heat trace as

$$
\mathrm{S} \operatorname{Tr} K_{M}(t)=\mathrm{H} \operatorname{Tr} K_{M}(t)+\operatorname{vol}(M) K_{\mathbf{h}}(t, 0) .
$$

In the case $M$ is a compact Riemann surface, the regularized heat trace is simply the trace of the heat kernel. If $M$ is a hyperbolic Riemann surface of finite volume but not connected, let $M_{1}, \cdots, M_{n}$ be the connected components, and define

$$
\mathrm{H} \operatorname{Tr} K_{M}(t)=\sum_{j=1}^{n} \mathrm{H} \operatorname{Tr} K_{M_{j}}(t) \quad \text { and } \quad \mathrm{S} \operatorname{Tr} K_{M}(t)=\sum_{j=1}^{n} \mathrm{~S} \operatorname{Tr} K_{M_{j}}(t) .
$$

The following result due to Selberg [Se] evaluates the integral representation stated in Theorem 1.1(b).

Theorem 1.3. Let $M$ be a connected, hyperbolic Riemann surface of finite volume with $p$ cusps. Let $H(\Gamma)$ denote a set of representatives of inconjugate primitive hyperbolic classes of a uniformizing group $\Gamma$ of $M$. Let $\ell(\gamma)$ be the length of the geodesic in the homotopy class determined by $\gamma \in \Gamma$, so then $\operatorname{Tr}(\ell(\gamma))=$ $2 \sinh (\ell(\gamma) / 2)$. Then

$$
\operatorname{HTr} K_{M}(t)=\frac{e^{-t / 4}}{(16 \pi t)^{1 / 2}} \sum_{n=1}^{\infty} \sum_{H(\Gamma)} \frac{\ell(\gamma)}{\sinh (n \ell(\gamma) / 2)} e^{-(n \ell(\gamma))^{2} / 4 t} .
$$

The proof of Theorem 1.3 follows directly from Theorem 1.1(b) and the following proposition, which is due to Selberg [Se] (see also [He2] and [M]). For the sake of completeness, we repeat the proof from $[\mathrm{M}]$.

Proposition 1.4. For any $q>0$, let $\gamma$ be a hyperbolic element with

$$
|\operatorname{Tr}(\gamma)|=2 \sinh (q / 2)>0,
$$


and let $C_{q}=\langle\gamma\rangle \backslash \mathbf{h}$. Then for any $t>0$, we have

$$
\int_{C_{q}}\left(K_{C_{q}}-K_{\mathbf{h}}\right)(t, x, x) d \mu(x)=\frac{q \exp (-t / 4)}{(4 \pi t)^{1 / 2}} \sum_{n=1}^{\infty} \frac{\exp \left(-(n q)^{2} / 4 t\right)}{\sinh (n q / 2)} .
$$

Proof. We model the domain of integration by two copies of the region

$$
\{\rho \exp (i \alpha): \rho \in(1, \exp (q)], \quad \alpha \in(0, \pi / 2) \cdot\} .
$$

With respect to these coordinates, the hyperbolic metric has volume element equal to $\rho^{-1} \sin (\alpha)^{-2} d \alpha d \rho$. Let

$$
a(q, n)=d_{\mathbf{h}}\left(\rho e^{i \alpha}, \rho e^{n q} e^{i \alpha}\right),
$$

where $d_{\mathbf{h}}$ is hyperbolic distance. From page 130 of [Be] we have, for any fixed $\rho>1$ the formula

$$
\cosh (a(q, n))=1+\frac{2 \sinh ^{2}(n q / 2)}{\sin ^{2} \alpha} .
$$

Notice that $a(q, n)$ depends on $\alpha$ but is independent of $\rho$. Therefore, we can integrate with respect to $\rho$, yielding the equality

$$
\begin{aligned}
\int_{C_{q}}\left(K_{C_{q}}-K_{\mathbf{h}}\right)(t, x, x) d \mu(x) & =4 \sum_{n=1}^{\infty} \int_{0}^{\pi / 2} \int_{1}^{\exp (q)} K_{\mathbf{h}}(t, a(q, n)) \frac{d \rho d \alpha}{\rho \sin ^{2} \alpha} \\
& =4 q \int_{0}^{\pi / 2} \sum_{n=1}^{\infty} K_{\mathbf{h}}(t, a(q, n)) \frac{d \alpha}{\sin ^{2} \alpha} .
\end{aligned}
$$

We shall evaluate each term in the sum over $n$ in (1.5); specifically, we can use the integral expression for the hyperbolic heat kernel to write

$$
\begin{aligned}
& 4 q \int_{0}^{\pi / 2} K_{\mathbf{h}}(t, a(q, n)) \frac{d \alpha}{\sin ^{2} \alpha} \\
& \quad=\frac{4 q \sqrt{2} \exp (-t / 4)}{(4 \pi t)^{3 / 2}} \int_{0}^{\pi / 2} \int_{a(q, n)}^{\infty} \frac{\beta \exp \left(-\beta^{2} / 4 t\right) d \beta d \alpha}{[\cosh \beta-\cosh (a(q, n))]^{1 / 2} \sin ^{2} \alpha} .
\end{aligned}
$$

Let $\sin ^{2} \alpha=u^{-1}$ so then

$$
\cosh a(q, n)=1+2 u \sinh ^{2}(n q / 2),
$$

and write (1.6) as

$$
=\frac{2 q \sqrt{2} \exp (-t / 4)}{(4 \pi t)^{3 / 2}} \int_{1}^{\infty} \int_{a(q, n)}^{\infty} \frac{\beta \exp \left(-\beta^{2} / 4 t\right) d \beta d u}{\left[\cosh \beta-1-2 u \sinh ^{2}(n q / 2)\right]^{1 / 2}(u-1)^{1 / 2}} .
$$


Define

$$
d(q, n)=\frac{\cosh \beta-1}{2 \sinh ^{2}(n q / 2)} .
$$

Notice that $d(q, n)$ depends on the variable $\beta$. If we interchange the order of integration in (1.7), we can write (1.7) as

$$
=\frac{2 q \sqrt{2} \exp (-t / 4)}{(4 \pi t)^{3 / 2}} \int_{n q}^{\infty} \int_{1}^{d(q, n)} \frac{\beta \exp \left(-\beta^{2} / 4 t\right) d u d \beta}{\left[\cosh \beta-1-2 u \sinh ^{2}(n q / 2)\right]^{1 / 2}(u-1)^{1 / 2}} .
$$

The inner integral now can be evaluated. Indeed, from the basic formula

$$
\int \frac{d x}{\sqrt{a+b x-x^{2}}}=\cos ^{-1}\left(\frac{b-2 x}{\sqrt{b^{2}+4 a}}\right)+C
$$

we obtain

$$
\int_{1}^{d(q, n)} \frac{d u}{[\cosh \beta-\cosh (a(q, n))]^{1 / 2}(u-1)^{1 / 2}}=\frac{\pi}{\sqrt{2} \sinh (n q / 2)} .
$$

Therefore, we have

$$
\begin{aligned}
4 q \int_{0}^{\pi / 2} K_{\mathbf{h}}(t, a(q, n)) \frac{d \alpha}{\sin ^{2} \alpha} & =\frac{2 \pi q \exp (-t / 4)}{(4 \pi t)^{3 / 2} \sinh (n q / 2)} \int_{n q}^{\infty} \beta \exp \left(-\beta^{2} / 4 t\right) \\
& =\frac{q \exp (-t / 4)}{(4 \pi t)^{1 / 2} \sinh (n q / 2)} \exp \left(-(n q)^{2} / 4 t\right)
\end{aligned}
$$

which gives the result.

Remark 1.5. If $M$ is a compact surface, then the regularized trace of the heat kernel is simply

$$
\mathrm{S} \operatorname{Tr} K_{M}(t)=\operatorname{Tr} K_{M}(t)=\sum_{n=0}^{\infty} e^{-\lambda_{n} t}
$$

where $\left\{\lambda_{n}\right\}$ is the set of eigenvalues of the Laplacian which acts on the space of smooth functions on $M$. Let $\left\{r_{n}\right\}$ be the set of numbers for which $1 / 4+r_{n}^{2}=\lambda_{n}$. The above calculation establishes then the formula

$$
\begin{aligned}
\sum_{n=0}^{\infty} e^{-r_{n}^{2} t}= & \frac{1}{2 \pi} \int_{0}^{\infty} e^{-r^{2} t} \tanh (\pi r) r d r \\
& +\frac{1}{(16 \pi t)^{1 / 2}} \sum_{n=1}^{\infty} \sum_{H(\Gamma)} \frac{\ell(\gamma)}{\sinh (n \ell(\gamma) / 2)} e^{-(n \ell(\gamma))^{2} / 4 t}
\end{aligned}
$$


This formula agrees perfectly with that on page 42 of [He2]. In other words, we have established the formula

$$
\begin{aligned}
\sum_{n=0}^{\infty} f_{t}\left(r_{n}\right) & =\frac{1}{2 \pi} \int_{0}^{\infty} f_{t}(r) \tanh (\pi r) r d r \\
& +\sum_{n=1}^{\infty} \sum_{H(\Gamma)} \frac{2 \ell(\gamma)}{\sinh (n \ell(\gamma) / 2)} \hat{f}_{t}(n \ell)
\end{aligned}
$$

where $f_{t}(x)=e^{-t x^{2}}$ and $\hat{f}_{t}$ denotes Fourier transform. By linearity and an elementary compuation, one shows that (1.9) then holds for any function $f$ of the form

$$
f(x)=\sum_{n=1}^{N} p_{n}\left(x^{2}\right) e^{-x^{2} t_{n}}
$$

where $t_{n}>0$ and $p_{n}$ is a polynomial. From this, and an application of the StoneWeierstrass theorem, one obtains the Selberg trace formula for compact hyperbolic Riemann surfaces (see page 32 of [He2] and references therein). Further details and discussion of this point of view is given in [HJL2].

Remark 1.6. If $M$ is non-compact, then it is much harder to give a spectral representation of the regularized heat trace (1.8). Various references exist for such a calculation, and the end result is the formula

$$
\begin{aligned}
\mathrm{S} T r K_{M}(t) & =\sum_{C(M)} e^{-\lambda_{n} t}-\frac{1}{4 \pi} \int_{-\infty}^{\infty} e^{-r^{2} t} \phi^{\prime} / \phi(1 / 2+i r) d r \\
& +\frac{p}{2 \pi} \int_{-\infty}^{\infty} e^{-r^{2} t} \Gamma^{\prime} / \Gamma(1+i r) d r-\frac{1}{4}(p-\operatorname{Tr} \Phi(1 / 2))+\frac{p \log 2}{\sqrt{4 \pi t}}
\end{aligned}
$$

where $C(M)$ denotes the (possibly finite) set of eigenvalues corresponding to $L^{2}$ eigenfunctions on $M$, and $\phi(s)$ is the determinant of the scattering matrix $\Phi(s)$. For further discussion, the reader is referred to [Se], [He3], or [Mü].

\section{$\S 2$. A degenerating heat trace}

In this section, we will consider the behavior of regularized heat traces on a degenerating family of hyperbolic Riemann surfaces. Based on Proposition 1.4 above, we have the following proposition. 
Proposition 2.1. Let $M_{\ell}$ denote a degenerating family of compact or non-compact hyperbolic Riemann surfaces of finite volume which converges to the non-compact hyperbolic surface $M_{0}$. Define the degenerating heat trace as the integral

$$
\operatorname{DTr} K_{M_{\ell}}(t)=\frac{1}{2} \int_{\cup C_{\ell}}\left[K_{C_{\ell}}(x, x, t)-K_{\mathbf{h}}(0, t)\right] d \mu(x) .
$$

Then for any $t>0$ we have the equality

$$
\mathrm{D} \operatorname{Tr} K_{M_{\ell}}(t)=\frac{e^{-t / 4}}{(16 \pi t)^{1 / 2}} \sum_{n=1}^{\infty} \sum_{D H(\Gamma)} \frac{\ell(\gamma)}{\sinh (n \ell(\gamma) / 2)} e^{-(n \ell(\gamma))^{2} / 4 t}
$$

Proof. This is a direct application of Proposition 1.4.

The following theorem, which we quote from [JLu3], asserts that given a degenerating family of hyperbolic Riemann surfaces of finite volume, the hyperbolic heat trace minus the degenerating heat trace converges pointwise to the hyperbolic heat trace on the limit surface.

Theorem 2.2. Let $M_{\ell}$ denote a degenerating family of compact or non-compact hyperbolic Riemann surfaces of finite volume which converges to the non-compact hyperbolic surface $M_{0}$. Then for any fixed $t>0$, we have the limit

$$
\lim _{\ell \rightarrow 0}\left[\mathrm{H} \operatorname{Tr} K_{M_{\ell}}(t)-\mathrm{D} \operatorname{Tr} K_{M_{\ell}}(t)\right]=\mathrm{H} \operatorname{Tr} K_{M_{0}}(t) .
$$

Outline of Proof. If $M_{\ell}$ is a degenerating family of compact surfaces, we can write

$$
\begin{aligned}
\operatorname{HTr} K_{M_{\ell}}(t)-\mathrm{D} \operatorname{Tr} K_{\ell}(t) & =\int_{M_{\ell} \backslash C_{\ell, \varepsilon}}\left[K_{M_{\ell}}(t, x, x)-K_{\mathbf{h}}(t, 0)\right] d \mu(x) \\
& +\int_{C_{\ell, \varepsilon}}\left[K_{M_{\ell}}(t, x, x)-K_{C_{\ell}}(t, x, x)\right] d \mu(x) \\
& -\int_{C_{\ell} \backslash C_{\ell, \varepsilon}}\left[K_{C_{\ell}}(t, x, x)-K_{\mathbf{h}}(t, 0)\right] d \mu(x) .
\end{aligned}
$$

Integral (I) converges to the corresponding integral over the limit surface $M_{0}$ by the heat kernel convergence theorem proved in [JLu1] (see also [JLu2] and [JLu3]). As for integral (II), one applies the maximum priniciple as in the proof of Theorem 1.1. Finally, for integral (III), one uses the bound (1.2), which implies

$$
\lim _{\varepsilon \rightarrow \infty} \int_{\cup C_{j, \varepsilon}}\left[K_{M}(t, x, x)-K_{C_{0}}(t, x, x)\right] d \mu(x)=0,
$$


together with a heat kernel convergence theorem for the heat kernel on $C_{q}$ over the region $C_{q, \delta} \backslash C_{q, \varepsilon}$ for any $0<\varepsilon<\delta<\infty$.

If $M_{\ell}$ is a degenerating family of non-compact surfaces, then one needs to regularize the heat traces near the existing cusps on each $M_{\ell}$. Specifically, one has the expression

$$
\begin{aligned}
\operatorname{HTr} K_{M_{\ell}}(t)-\operatorname{DTr} K_{\ell}(t) & =\int_{M_{\ell} \backslash\left(C_{\ell, \varepsilon} \cup C_{0, \varepsilon}\right)}\left[K_{M_{\ell}}(t, x, x)-K_{\mathbf{h}}(t, 0)\right] d \mu(x) \\
& +\int_{C_{0, \varepsilon}}\left[K_{M_{\ell}}(t, x, x)-K_{C_{0}}(t, x, x)\right] d \mu(x) \\
& -\int_{C_{0} \backslash C_{0, \varepsilon}}\left[K_{C_{0}}(t, x, x)-K_{\mathbf{h}}(t, 0)\right] d \mu(x) \\
& +\int_{C_{\ell, \varepsilon}}\left[K_{M_{\ell}}(t, x, x)-K_{C_{\ell}}(t, x, x)\right] d \mu(x) \\
& -\int_{C_{\ell} \backslash C_{\ell, \varepsilon}}\left[K_{C_{\ell}}(t, x, x)-K_{\mathbf{h}}(t, 0)\right] d \mu(x) .
\end{aligned}
$$

We have labeled two integrals by (II) and two integrals by (III) since these terms are formally identical and will be handled by similarly methods in further arguments. The reader is referred to [JLu3] for further details.

Corollary 2.3. Let $M_{\ell}$ denote a degenerating family of compact or non-compact hyperbolic Riemann surfaces of finite volume which converges to the non-compact hyperbolic surface $M_{0}$. Then for fixed $\delta>0$, there is a positive $c$ such that for all $t<\delta$

$$
\operatorname{HTr} K_{M_{\ell}}(t)-\operatorname{DTr} K_{M_{\ell}}(t)=O\left(e^{-c / t}\right)
$$

uniformly in $\ell$.

Proof. The uniform convergence of heat kernels from [JLu1] and [JLu3] implies that integral (I) has exponential decay as $t \rightarrow 0$, uniformly in $\ell$. By applying the maximum principle, as in the proof of Theorem 1.1 , one shows that the asymptotic behavior of integral (II) as $t \rightarrow 0$ is that of exponential decay, uniformly in $\ell$. As in the proof of Theorem 2.2, we can write integral (III) as a sum of two integrals, one for which the bound (1.2) implies exponential decay and the other over a compact region for which one has uniform convergence of heat kernels, we conclude that integral (III) has exponential decay as $t \rightarrow 0$, uniformly in $\ell$. Combining these bounds, the stated result is proved.

In summary, Theorem 2.2 proves that the hyperbolic heat trace minus the degenerating heat trace converges pointwise to the hyperbolic heat trace. Since the 
volume of the hyperbolic Riemann surfaces remains constant through degeneration, one immediately has that the regularized heat trace minus the degenerating heat trace converges pointwise to the regularized heat trace on the limit surface. Corollary 2.3 states that the proof of Theorem 2.2 provides uniformity of the asymptotics near $t=0$ of the hyperbolic heat trace minus the degenerating heat trace. What remains to consider is the uniformity of the asymptotics for all $t \in \mathbf{R}^{+}$.

\section{$\S 3$. Uniform long time asymptotics}

In this section we give a further analysis of the asymptotics of the uniformity of the pointwise convergence in Theorem 2.1 for values of $t$ near infinity. The main result of this section is the following theorem.

Theorem 3.1. Let $M_{\ell}$ denote a degenerating family of compact or non-compact hyperbolic Riemann surfaces of finite volume which converges to the non-compact hyperbolic surface $M_{0}$. Let $0 \leq \alpha<1 / 4$ be such that $\alpha$ is not an eigenvalue of $M_{0}$. Let

$$
\operatorname{HTr} K_{M_{\ell}}^{(\alpha)}(t)=\operatorname{HTr} K_{M_{\ell}}(t)-\sum_{\lambda_{n, \ell} \leq \alpha} e^{-\lambda_{n, \ell} t} .
$$

Then for any $c<\alpha$, there exists a constant $C$ such that the bound

$$
\left|\mathrm{H} \operatorname{Tr} K_{M_{\ell}}^{(\alpha)}(t)-\mathrm{D} \operatorname{Tr} K_{\ell}(t)\right| \leq C \exp (-c t)
$$

holds for all $t \geq 0$ and uniformly in $\ell$.

Our proof of Theorem 3.1 comes from analyzing the three integrals in Theorem 2.2. For the integral over the portion the surfaces $M_{\ell}$ and $C_{\ell}$ away from the developing cusps, we need the following lemma.

Lemma 3.2. Let $R_{\ell}$ denote either $M_{\ell}$ or $C_{\ell}$, that is, either a degenerating hyperbolic surface of finite volume or a degenerating infinite hyperbolic cylinder. For any $\alpha<1 / 4$ and $c<\alpha$, the limit

$$
\lim _{\ell \rightarrow 0} \exp (c t) K_{R_{\ell}}^{(\alpha)}(t, x, x)=\exp (c t) K_{R_{0}}^{(\alpha)}(t, x, x)
$$

is uniform for $x \in R_{\ell} \backslash C_{\ell, \varepsilon}$ and $t>0$.

Proof. We shall argue as in the proof of Theorem 1(b) of [JLu1]. From the spectral measure, we can express the heat kernel via the integral

$$
K_{R_{\ell}}^{(\alpha)}(t, x, y)=\int_{\alpha}^{\infty} \exp (-\lambda t) d N_{R_{\ell}}(x, y ; \lambda) .
$$


Observe that $d N_{R_{\ell}}(x, x ; \lambda)$ is a positive measure. Let $t=t_{0}+s$ and write

$$
\begin{aligned}
0 \leq K_{R_{\ell}}^{(\alpha)}(t, x, x) & =\int_{\alpha}^{\infty} \exp \left(-\lambda t_{0}\right) \exp (-\lambda s) d N_{R_{\ell}}(x, y ; \lambda) \\
& \leq \exp (-\alpha s) \int_{\alpha}^{\infty} \exp \left(-\lambda t_{0}\right) d N_{R_{\ell}}(x, y ; \lambda) \\
& \leq \exp (-\alpha s) K_{R_{\ell}}^{(\alpha)}\left(t_{0}, x, x\right) \\
& =\exp (-\alpha t) \exp \left(\alpha t_{0}\right) K_{R_{\ell}}^{(\alpha)}\left(t_{0}, x, x\right) ;
\end{aligned}
$$

hence the quantity

$$
0 \leq \exp (\alpha t) K_{R_{\ell}}^{(\alpha)}(t, x, x)
$$

is monotone decreasing in $t$. From Theorem 1.3 of [JLu3] and the convergence of small eigenvalues and small eigenfunctions (see [CC], [He1] or [HJL1]), we know that $K_{\ell}^{(\alpha)}\left(t_{0}, x, x\right)$ converges uniformly to $K_{0}^{(\alpha)}\left(t_{0}, x, x\right)$ for $x \in R_{\ell} \backslash C_{\ell, \varepsilon}$. Therefore, for $t \geq t_{0}$, there is a constant $C=C\left(\varepsilon, t_{0}\right)$ which is independent of $\ell$ such that

$$
\exp (\alpha t) K_{R_{\ell}}^{(\alpha)}(t, x, x) \leq C .
$$

If $c<\alpha$, then

$$
\exp (c t) K_{R_{\ell}}^{(\alpha)}(t, x, x) \leq C \exp (t(c-\alpha))
$$

We now can combine the monotonicity and pointwise convergence of the function $\exp (\alpha t) K_{R_{\ell}}^{(\alpha)}(t, x, x)$ to $\exp (\alpha t) K_{R_{0}}^{(\alpha)}(t, x, x)$, as in the proof of Theorem $1(\mathrm{~b})$ from [JLu1], to finish the proof.

Lemma 3.3. Let $M_{\ell}$ denote a degenerating family of compact or non-compact hyperbolic Riemann surfaces of finite volume which converges to the non-compact hyperbolic surface $M_{0}$. Let $\alpha<1 / 4, c<\alpha$, and $\delta<1 / 2$. Then there is a constant $C$ such that for all $t>0$ we have

$$
\sup _{\substack{\ell>0 \\ \zeta \in \partial C_{\ell, \delta} \\ \xi \in \partial C_{\ell, \delta}}}\left|K_{M_{\ell}}^{(\alpha)}(t, \zeta, \xi)-K_{C_{\ell}}(t, \zeta, \xi)\right| \leq C \exp (-c t) .
$$

Proof. As stated in the introduction, results from [R] allow us to take any $\delta<1 / 2$. With this, the claim follows directly from the bounds given in (1.3) and (1.4), to which we can apply Lemma 3.2 to both the upper bound and the lower bound, together with the observation that $1 / 4$ is the bottom of the spectrum for $C_{\ell}$.

Lemma 3.4. Let $f(t, x)$ be a solution to the Dirichlet heat problem on the finite cylinder $C_{\ell, \delta}$, and, for fixed $t>0$, let $\|f(t, \cdot)\|_{C_{\ell, \delta}, 2}$ denote the $L^{2}$ norm of $f(t, \cdot)$ as a function on $C_{\ell, \delta}$. Then for all $t_{0}, t>0$, we have

$$
\left\|f\left(t_{0}+t, \cdot\right)\right\|_{C_{\ell, \delta}, 2} \leq\left\|f\left(t_{0}, \cdot\right)\right\|_{C_{\ell, \delta}, 2} \cdot \exp (-t / 4)
$$


Proof. Directly from definitions, we have

$$
\partial_{t}\left\|f\left(t_{0}+t, \cdot\right)\right\|_{C_{\ell, \delta}, 2}^{2}=\int_{C_{\ell, \delta}} 2 f f_{t}=\int_{C_{\ell, \delta}} 2 f \Delta f=-2 \int_{C_{\ell, \delta}}|\nabla f|^{2} .
$$

Therefore,

$$
\begin{aligned}
\partial_{t}\left\|f\left(t_{0}+t, \cdot\right)\right\|_{C_{\ell, \delta}, 2}^{2} & =\left(\frac{-2 \int_{C_{\ell, \delta}}|\nabla f|^{2}}{\left\|f\left(t_{0}+t, \cdot\right)\right\|_{C_{\ell, \delta}, 2}^{2}}\right)\left\|f\left(t_{0}+t, \cdot\right)\right\|_{C_{\ell, \delta}, 2}^{2} \\
& \leq-\frac{1}{2}\left\|f\left(t_{0}+t, \cdot\right)\right\|_{C_{\ell, \delta}, 2}^{2}
\end{aligned}
$$

The result follows from integration.

To finish the background material necessary for our proof of Theorem 3.1, we need a basic proposition from [JLu3] for the Poisson kernel on $C_{\ell, \varepsilon}$. For completeness, let us recall the definition of the Poisson kernel together with a result from [JLu3]. After this, the proof of Theorem 3.1 will be given.

Definition 3.5. Let $K_{C_{q, \delta}}^{D}(t, x, y)$ be the Dirichlet heat kernel on the domain $C_{q, \delta}$. For any $\zeta \in \partial C_{q, \delta}$, let $\partial_{n, \zeta}$ denote the inward normal derivative. The Poisson kernel $P_{q, \delta}(t, x, \zeta)$ of the domain $C_{q, \delta}$ is defined to be

$$
P_{q, \delta}(t, x, \zeta)=\partial_{n, \zeta} K_{C_{q, \delta}}^{D}(t, x, \zeta)
$$

Remark 3.6. From Theorem 5 on page 168 of $[\mathrm{Ch}]$, we have the following characterization of the Poisson kernel. The function $P_{q, \delta}(t, x, \zeta)$ is an integral kernel for $t>0$ with $x \in C_{q, \delta}$ and $\zeta \in \partial C_{q, \delta}$, which solves the following boundary value problem. Let $u=u(t, x)$ satisfy

$$
\left(\Delta-\partial_{t}\right) u=0, \quad u(0, x)=0, \quad \text { and } \quad u(t, \zeta)=f(t, \zeta) \quad \text { for } \zeta \in \partial C_{q, \delta} .
$$

Then

$$
u(t, x)=\int_{0}^{t} \int_{\partial C_{q, \delta}} P_{q, \delta}(t-\sigma, x, \zeta) f(\sigma, \zeta) d \varrho(\zeta) d \sigma .
$$

The following proposition, which we quote from [JLu3], establishes various estimates for the Poisson kernel which are independent of $q$.

Proposition 3.7. Let $C_{q}$ be a family of infinite volume hyperbolic cylinders. For any $\delta>0$, any $0<\varepsilon<\delta$, and any real numbers $t_{0}, t_{1}>0$, the following results hold. 
a) For all $0<t \leq t_{1}, x \in C_{q, \varepsilon}$ and $\zeta \in \partial C_{q, \delta}$, there is a constant $C$ independent of $q$ such that

$$
0 \leq P_{q, \delta}(t, x, \zeta) \leq C
$$

b) For all $0<t_{0} \leq t \leq t_{1}, x \in C_{q, \delta}$ and $\zeta \in \partial C_{q, \delta}$, there is a constant $C$ independent of $q$ such that

$$
0 \leq P_{q, \delta}(t, x, \zeta) \leq C
$$

c) For fixed $s$, the $L^{2}$ norm $\left\|P_{q, \delta}(t+i s, \cdot, \zeta)\right\|_{C_{q, \delta}, 2}$ is decreasing in $t$.

By combining Lemma 3.4 and Proposition 3.7, we obtain the following bound.

Lemma 3.8. For any $\varepsilon<\delta$, there is a constant $C$ such that

$$
\left\|P_{\ell, \delta}(t, \zeta, \cdot)\right\|_{C_{\ell, \varepsilon}, 2} \leq C \exp (-t / 4) .
$$

Proof. Pick any $t_{0}$. If $t<t_{0}$, then Proposition 3.7 provides a supremum bound which is uniform in $\ell$, hence we have a bound on the $L^{2}$ norm, namely

$$
\sup _{\substack{\zeta \in \partial C_{\ell, \delta} \\ x \in C_{\ell, \varepsilon}}}\left|P_{\ell, \delta}\left(t_{0}, \zeta, x\right)\right| \leq c\left(t_{0}\right) .
$$

In fact, since the variables $\zeta$ and $x$ are bounded apart, one can take $t_{0}=0$. For $t>t_{0}$, apply Lemma 3.4 to get

$$
\left\|P_{\ell, \delta}(t, \zeta, \cdot)\right\|_{C_{\ell, \varepsilon}, 2} \leq\left\|P_{\ell, \delta}(t, \zeta, \cdot)\right\|_{C_{\ell, \delta}, 2} \leq\left\|P_{\ell, \delta}\left(t_{0}, \zeta, \cdot\right)\right\|_{C_{\ell, \delta}, 2} \cdot c\left(t_{0}\right) e^{-t / 4},
$$

which holds since $\lambda_{1} \geq 1 / 4$. Combining the inequalities obtained in the two cases, the asserted result is established.

Proof of Theorem 3.1. The uniformity as asserted for integral (III) is given by (1.2). Lemma 3.2 applies to integral (I), so it remains to consider integral (II). For this, we need the above lemmas and argue as follows.

Let $\left\{\lambda_{n, \ell}\right\}$ be the family of eigenvalues on $M_{\ell}$ which converge to the eigenvalues on $M_{0}$ which are less than $1 / 4$, and let $\left\{\phi_{n, \ell}(x)\right\}$ be the associated family of eigenfunctions. By results from [CC], [He1] or [HJLu1], the sum

$$
\sum_{\lambda_{n, \ell}<1 / 4} e^{-t \lambda_{n, \ell}} \phi_{n, \ell}(x) \phi_{n, \ell}(y)
$$

varies continuously in $\ell$ up to and including $\ell=0$. Let $\delta<1 / 2$ be fixed, and let $0<\varepsilon<\delta$. For $x, y \in C_{\ell, \delta}$ and $t>0$, consider the decomposition

$$
K_{M_{\ell}}(t, x, y)-K_{C_{\ell}}(t, x, y)=u(t, x, y)+v(t, x, y)+\sum_{n=1}^{N} e^{-t \lambda_{n, \ell}} \phi_{n, \ell}(x) \phi_{n, \ell}(y)
$$


where $u$ and $v$ are solutions to the homogeneous heat equation in both $x$ and $y$ (and $t$ ) such that:

- $u$ has values identically zero on $\partial C_{\ell, \delta}$ and has appropriate initial values;

- $v$ has identically zero initial values but has appropriate boundary values on $\partial C_{\ell, \delta}$.

Let $\alpha<1 / 4$ be such that $M_{0}$ has no eigenvalues in the range $(\alpha, 1 / 4)$. With the above decomposition, we have

$$
K_{M_{\ell}}^{(\alpha)}(t, x, y)-K_{C_{\ell}}(t, x, y)=u(t, x, y)+v(t, x, y) .
$$

We shall study the functions $u(t, x, y)$ and $v(t, x, y)$ separately.

From two applications of the Poisson kernel, we have the expression

$v(t, x, y)=\int_{0}^{t} \int_{\partial C_{\ell, \delta}} \int_{0}^{\tau} \int_{\partial C_{\ell, \delta}} P_{\ell, \delta}(t-\tau, x, \zeta) P_{\ell, \delta}(\tau-\sigma, y, \xi) D_{\ell}^{(\alpha)}(\sigma, \zeta, \xi) d \xi d \sigma d \zeta d \tau$

where

$$
D_{\ell}^{(\alpha)}(\sigma, \zeta, \xi)=K_{M_{\ell}}^{(\alpha)}(\sigma, \zeta, \xi)-K_{C_{\ell}}(\sigma, \zeta, \xi) .
$$

We need to consider the integral

$$
\int_{C_{\ell, \varepsilon}} v(t, x, x) d \mu(x)
$$

If we use the sup-norm on difference of heat kernels, as given in Lemma 3.3, and the $L^{2}$ norm of the Poisson kernel, as given in Lemma 3.8, together with the Cauchy-Schwarz inequality, we obtain the bound

$$
\int_{C_{\ell, \varepsilon}} v(t, x, x) d \mu(x) \leq C \int_{0}^{t} \int_{0}^{\tau} \exp (-(t-\tau) / 4) \exp (-(\tau-\sigma) / 4) \exp (-c \sigma) d \sigma d \tau,
$$

which is easily shown to satisfy the bound $O\left(e^{-c t}\right)$.

It remains to consider the $L^{2}$-norm of $u(t, x, x)$ over $C_{\ell, \varepsilon}$. Let $N$ be an integer which bounds the number of eigenvalues on each $M_{\ell}$ less than $1 / 4$; such a universal choice of $N$ is possible by Buser's theorem (see page 251 of $[\mathrm{Ch}]$ ). As a function of $x$ and $t$ with $y$ fixed, $u(t, x, y)$ satisfies the heat equation with zero boundary data and initial data given by

$$
g(x, y)=\sum_{\lambda_{n, \ell}<1 / 4} \phi_{n, \ell}(x) \phi_{n, \ell}(y) .
$$

Therefore, we can write

$$
u(t, x, y)=\int_{C_{\ell, \delta}} K_{\ell, \delta}^{D}(t, z, x) g(z, y) d \mu(z) .
$$


Now view (3.2) as a solution to the heat equation on $C_{\ell, \delta}$ in the variables $y$ and $t$ with $x$ fixed. This yields the expression

$$
\begin{aligned}
u(\tau+t, x, y) & =\int_{C_{\ell, \delta}} K_{\ell, \delta}^{D}(\tau, w, y) u(t, x, w) d \mu(w) \\
& =\int_{C_{\ell, \delta}} K_{\ell, \delta}^{D}(\tau, w, y)\left(\int_{C_{\ell, \delta}} K_{\ell, \delta}^{D}(t, z, x) g(z, w) d \mu(z)\right) d \mu(w) .
\end{aligned}
$$

Hence,

$$
\begin{aligned}
& u(\tau+t, x, y)=\sum_{\lambda_{n, \ell}<1 / 4} \int_{C_{\ell, \delta}} \int_{C_{\ell, \delta}} K_{\ell, \delta}^{D}(\tau, w, y) K_{\ell, \delta}^{D}(t, z, x) \phi_{n, \ell}(z) \phi_{n, \ell}(w) d \mu(z) d \mu(w) \\
& =\sum_{\lambda_{n, \ell}<1 / 4}\left(\int_{C_{\ell, \delta}} K_{\ell, \delta}^{D}(\tau, w, y) \phi_{n, \ell}(w) d \mu(w)\right)\left(\int_{C_{\ell, \delta}} K_{\ell, \delta}^{D}(t, z, x) \phi_{n, \ell}(z) d \mu(z)\right) .
\end{aligned}
$$

If we set $x=y$ and $t=\tau$, and change the variable in the second integral in (3.4) from $w$ to $z$, we get

$$
u(2 t, x, x)=\sum_{\lambda_{n, \ell}<1 / 4}\left(\int_{C_{\ell, \delta}} K_{\ell, \delta}^{D}(t, z, x) \phi_{n, \ell}(z) d \mu(z)\right)^{2} .
$$

Now write

$$
\sum_{m=1}^{\infty} a_{n, m} e^{-\lambda_{m} t} \psi_{m}(x)=\int_{C_{\ell, \delta}} K_{C_{\ell, \delta}}^{D}(t, x, z) \phi_{n, \ell}(z) d \mu(z),
$$

where $\left\{\psi_{m}(x)\right\}$ is the orthonormal basis of eigenfunctions of the Dirichlet problem on $C_{\ell, \delta}$. The positivity of the expression (3.5) immediately allows us to conclude the inequality

$$
0 \leq F_{\varepsilon}(t)=\int_{C_{\ell, \varepsilon}} u(t, x, x) d \mu(x) \leq \int_{C_{\ell, \delta}} u(t, x, x) d \mu(x)=F_{\delta}(t),
$$

so it suffices to prove $F_{\delta}(t) \leq C \exp (-t / 4)$ for some constant $C$ which is independent of $\ell$. Notice that we have the equality

$$
F_{\delta}(t)=\int_{C_{\ell, \delta}} u(t, x, x) d \mu(x)=\sum_{\lambda_{n, \ell}<1 / 4} \sum_{m=1}^{\infty} a_{n, m}^{2} e^{-\lambda_{m} t} .
$$


From this formula, it is immediate that $F_{\delta}(t)$ is monotone decreasing in $t$. Further, from (3.5), it follows that $F_{\delta}(0) \leq N$. Since $\lambda_{m} \geq 1 / 4$ for all $m$, we have, from $(3.6), F_{\delta}^{\prime}(t) \leq(-1 / 4) F_{\delta}(t)$. If we integrate this inequality, we conclude

$$
F_{\delta}(t) \leq F_{\delta}(0) e^{-t / 4},
$$

from which we obtain

$$
0 \leq F_{\varepsilon}(t) \leq F_{\delta}(t) \leq F_{\delta}(0) e^{-t / 4} \leq N e^{-t / 4} .
$$

With this, the proof of Theorem 3.1 is complete.

\section{$\S 4$. Applications to spectral functions}

We shall now consider a few applications of our convergence theorem for the regularized heat trace and the truncated regularized heat trace. The two applications we consider involve the Mellin transform, which yields a generalization of the spectral zeta function, and the Laplace transform with a quadratic change of variables, which yields the Selberg zeta function.

For any $\alpha \in(0,1 / 4)$, let us define the zeta function

$$
\zeta_{M}^{(\alpha)}(s)=\frac{1}{\Gamma(s)} \int_{0}^{\infty} \mathrm{S} \operatorname{Tr} K_{M}^{(\alpha)}(t) t^{s} \frac{d t}{t} .
$$

If $M$ is compact and $\alpha<\lambda_{1}$, then

$$
\zeta_{M}^{(\alpha)}(s)=\sum_{0<\lambda_{n}} \lambda_{n}^{-s}=\frac{1}{\Gamma(s)} \int_{0}^{\infty}\left[\mathrm{S} \operatorname{Tr} K_{M}(t)-m_{0}(M)\right] t^{s} \frac{d t}{t},
$$

where $m_{0}(M)$ is equal to the number of connected components of $M$. Thus, our spectral zeta function generalizes the usual definition which applies only in the case $M$ is compact.

Theorem 4.1. Let $M_{\ell}$ denote a degenerating family of compact or non-compact hyperbolic Riemann surfaces of finite volume which converges to the non-compact hyperbolic surface $M_{0}$. Let $\alpha<1 / 4$ be any number that is not an eigenvalue of $M_{0}$. Then for any $s \in \mathbf{C}$, we have

$$
\lim _{\ell \rightarrow 0}\left[\zeta_{M_{\ell}}^{(\alpha)}(s)-\frac{1}{\Gamma(s)} \int_{0}^{\infty} \mathrm{D} \operatorname{Tr} K_{M_{\ell}}(t) t^{s} \frac{d t}{t}-\zeta_{M_{0}}^{(\alpha)}(s)\right]=0 .
$$


The convergence is uniform in any half-plane of the form $\operatorname{Re}(s)>C>-\infty$.

Proof. By definition, we are to prove

$$
\frac{1}{\Gamma(s)} \lim _{\ell \rightarrow 0} \int_{0}^{\infty}\left[\mathrm{S} \operatorname{Tr} K_{M_{\ell}}^{(\alpha)}(t)-\mathrm{D} \operatorname{Tr} K_{M_{\ell}}(t)-\mathrm{S} \operatorname{Tr} K_{M_{0}}^{(\alpha)}(t)\right] t^{s} \frac{d t}{t}=0 .
$$

Since $\operatorname{vol}\left(M_{0}\right)=\operatorname{vol}\left(M_{\ell}\right)$, it is equivalent to prove

$$
\lim _{\ell \rightarrow 0} \int_{0}^{\infty}\left[\operatorname{H} \operatorname{Tr} K_{M_{\ell}}^{(\alpha)}(t)-\mathrm{D} \operatorname{Tr} K_{M_{\ell}}(t)-\mathrm{H} \operatorname{Tr} K_{M_{0}}^{(\alpha)}(t)\right] t^{s} \frac{d t}{t}=0 .
$$

By the results established in Theorem 2.2, Corollary 2.3, and Theorem 3.1, we can interchange the limit and integral, and the result follows.

Remark 4.2. By direct calculation, we have

$$
\int_{0}^{\infty} \operatorname{DTr} K_{M_{\ell}}(t) t^{s} \frac{d t}{t}=\sum_{n=1}^{\infty} \frac{\ell}{(16 \pi)^{1 / 2} \sinh (n \ell / 2)} K_{s-1 / 2}(1 / 2, n \ell / 2)
$$

where $K_{s}(a, b)$ is the $K$-Bessel function

$$
K_{s}(a, b)=\int_{0}^{\infty} e^{-a^{2} t-b^{2} / t} t^{s} \frac{d t}{t} .
$$

Since

$$
K_{1 / 2}(b, a)=K_{-1 / 2}(a, b)=\frac{\sqrt{\pi}}{b} e^{-2 a b},
$$

we have, in the special case $s=0$,

$$
\sum_{n=1}^{\infty} \frac{\ell}{(16 \pi)^{1 / 2} \sinh (n \ell / 2)} K_{-1 / 2}(1 / 2, n \ell / 2)=\sum_{n=1}^{\infty} \frac{e^{-n \ell}}{n\left(1-e^{-n \ell}\right)}
$$

(see [JLa2]). As a result, we have proved the following corollary.

Corollary 4.3. For any finite volume hyperbolic Riemann surface, define

$$
\log \operatorname{det}^{(\alpha)} \boldsymbol{\Delta}_{M}=-\partial_{s} \zeta_{M}^{(\alpha)}(0)
$$

Let $M_{\ell}$ denote a degenerating family of compact or non-compact hyperbolic Riemann surfaces of finite volume which converges to the non-compact hyperbolic 
surface $M_{0}$. Let $\alpha<1 / 4$ be a positive number such that $\alpha$ is not an eigenvalue of $M_{0}$. Then

$$
\lim _{\ell \rightarrow 0}\left[\log \operatorname{det}^{(\alpha)} \boldsymbol{\Delta}_{\ell}-\sum_{D H(\Gamma)} \sum_{n=1}^{\infty} \frac{e^{-n \ell}}{n\left(1-e^{-n \ell}\right)}-\sum_{\lambda_{n} \leq \alpha} \log \lambda_{n}\right]=\log \operatorname{det}^{(\alpha)} \boldsymbol{\Delta}_{0} .
$$

Proof. Since the gamma function has a first order pole at $s=0$, the degenerating term is as in (4.2).

Remark 4.4. The elementary lemma on page 112 of [He1] states, for $\operatorname{Re}(s)>0$, the asymptotic formula

$$
\sum_{D H(\Gamma)} \sum_{n=1}^{\infty} \frac{e^{-n s \ell}}{n\left(1-e^{-n \ell}\right)}=\sum_{D H(\Gamma)}\left(-\frac{\pi^{2}}{3 \ell}-(2 s-1) \log \ell\right)+O(1) .
$$

Therefore, Corollary 4.3 agrees with some of the main results from [He1] and [Wo] in the case $M_{\ell}$ is a degenerating family of compact surfaces. However, as stated above, the methods of proof in [He1] and [Wo] do not apply to the setting of a degenerating family of non-compact surfaces, whereas Corollary 4.3 includes these cases.

To continue, let us now apply the results from sections 2 and 3 to the Selberg zeta function. Recall that the logarithmic derivative of the Selberg zeta function is defined via the integral

$$
\frac{Z_{M}^{\prime}(s)}{Z_{M}(s)}=(2 s-1) \int_{0}^{\infty} \operatorname{HTr} K_{M}(t) e^{-s(s-1) t} d t
$$

From the collapse of the $K$-Bessel function (4.1), we have, following [JLa2], the evaluation

$$
\begin{gathered}
\frac{Z_{M}^{\prime}(s)}{Z_{M}(s)}=(2 s-1) \sum_{n=1}^{\infty} \sum_{\gamma \in H(\Gamma)} \frac{\ell(\gamma)}{(16 \pi)^{1 / 2} \sinh (n \ell(\gamma) / 2)} K_{1 / 2}(s-1 / 2, n \ell(\gamma) / 2) \\
=\sum_{n=1}^{\infty} \sum_{\gamma \in H(\Gamma)} \frac{\ell(\gamma)}{2 \sinh (n \ell(\gamma) / 2)} e^{-(s-1 / 2) n \ell(\gamma),}
\end{gathered}
$$

which agrees with Proposition 4.2 on page 67 of [He2]. The constant of integration obtained by integrating (4.3) is determined by defining, as on page 66 of [He2], the Selberg zeta function itself via the product

$$
Z_{M}(s)=\prod_{H(\Gamma)} \prod_{n=0}^{\infty}\left(1-e^{-(s+n) \ell(\gamma)}\right) .
$$


By an elementary argument involving hyperbolic geometry, one can estimate the asymptotic behavior of the number of closed geodesics of bounded length (see, for example, Lemma 4 of [JLu1] or Lemma 1.4 of [JLu3]). From this estimate, it is easy to show that the Euler product in (4.4) converges for $\operatorname{Re}(s)>1$.

For $\alpha<1 / 4$, let

$$
\frac{Z_{M}^{(\alpha)^{\prime}}(s)}{Z_{M}^{(\alpha)}(s)}=(2 s-1) \int_{0}^{\infty} \operatorname{HTr} K_{M}^{(\alpha)}(t) e^{-s(s-1) t} d t=\frac{Z_{M}^{\prime}(s)}{Z_{M}(s)}-\sum_{\lambda_{n} \leq \alpha} \frac{2 s-1}{s(s-1)-\lambda_{n}} .
$$

Theorem 4.5. Let $M_{\ell}$ denote a degenerating family of compact or non-compact hyperbolic Riemann surfaces of finite volume which converges to the non-compact hyperbolic surface $M_{0}$. Let $\alpha$ be such that $M$ has no eigenvalues in the range $[\alpha, 1 / 4)$. Then for any $s$ with $\operatorname{Re}(s)>1$ or in the region $\operatorname{Re}\left(s^{2}-s\right)>-1 / 4$, we have

$$
\lim _{\ell \rightarrow 0}\left[\frac{Z_{\ell}^{(\alpha)^{\prime}}(s)}{Z_{\ell}^{(\alpha)}(s)}-\sum_{n=1}^{\infty} \sum_{D H(\Gamma)} \frac{\ell(\gamma)}{2 \sinh (n \ell(\gamma) / 2)} e^{-(s-1 / 2) n \ell(\gamma)}\right]=\frac{Z_{0}^{(\alpha)^{\prime}}(s)}{Z_{0}^{(\alpha)}(s)}
$$

Proof. The proof of Theorem 4.5 for $s$ in the region $\operatorname{Re}\left(s^{2}-s\right)>-1 / 4$ follows the pattern of the proof of Theorem 4.1, which is a direct application of Theorem 2.2, Corollary 2.3, and Theorem 3.1. From (4.3), we have that the logarithmic derivative of the Selberg zeta function can be written as a Dirichlet series with positive coefficients in the region $\operatorname{Re}(s)>1$. Therefore, the convergence result extends from the region $\operatorname{Re}\left(s^{2}-s\right)>-1 / 4$, which contains the line segment $\mathbf{R}_{>1 / 2}$, to the $\operatorname{Re}\left(s^{2}-s\right)>-1 / 4$ together with the entire half plane $\operatorname{Re}(s)>1$.

Remark 4.6. As in the case of Theorem 4.1, the convergence result stated in Theorem 4.5 is related to results contained in [He1] and [Wo]. Our result applies to a slightly larger region than considered in [He1] or [Wo]. Also, as above, our work applies to degenerating non-compact surfaces as well as degenerating compact surfaces, whereas the techniques used in [He1] and [Wo] apply only in the compact setting. Also, note that if we restrict our attention to $\operatorname{Re}(s)>1$, then one can take $\alpha=0$.

Remark 4.7. The connection between the Selberg zeta function and the spectral zeta function is as follows. Let

$$
\zeta_{M}(w, z)=\frac{1}{\Gamma(s)} \int_{0}^{\infty}\left[\operatorname{STr} K_{M}^{(\alpha)}(t)-m_{0}(M)\right] e^{-t z} t^{w} \frac{d t}{t}
$$

which is defined for $\operatorname{Re}(z)$ sufficiently large. Following the proof of analytic continuation of Laplace-Mellin transform of theta functions, we have, at least formally, 
the formula

$$
\exp \left(-\partial_{w} \zeta_{M}(0, s(s-1))\right)=Z_{M}(s) F(s)^{2 g-2}
$$

where

$$
\begin{aligned}
F^{\prime} / F(s) & =(2 s-1)\left(\int_{0}^{\infty}\left[4 \pi K_{\mathbf{h}}(t, 0)-\frac{1}{t}\right] e^{-s(s-1)} d t-\log (s(s-1))\right. \\
& =(2 s-1) \int_{0}^{\infty}\left[4 \pi K_{\mathbf{h}}(t, 0)-\frac{e^{-t}}{t}\right] d t
\end{aligned}
$$

(see page 184 of [JLa1]). In [Sa] it was shown that (4.6) is a meromorphic function with simple poles and integer residues, hence the integral in (4.6) is indeed the $\operatorname{logarithmic}$ derivative of a meromorphic function $F$. Upon setting $s=1$ above, we have

$$
\log \operatorname{det}^{*} \boldsymbol{\Delta}_{M}=\log Z_{M}^{\prime}(1)+\frac{1}{2} \chi(M) c_{0} \quad \text { where } \quad c_{0}=-4 \zeta_{\mathbf{Q}}^{\prime}(-1)+\frac{1}{2}-\log (2 \pi) .
$$

The constant $c_{0}$ was evaluated in [Sa], which proved (4.5) and (4.7) for compact surfaces. The above analysis applies to establish (4.5) and (4.7) for all hyperbolic Riemann surfaces of finite volume.

\section{Acknowledgements}

The authors gratefully acknowledge many helpful conversations from Jonathan Huntley. We thank him for his assistance and encouragement. We also thank Jozek Dodziuk and Joseph Grotowski for comments made from a preliminary version of the manuscript.

\section{Bibliography}

[A1] W. Abikoff, The real analytic theory of Teichmüller space, Lecture Notes in Mathematics 820, Springer-Verlag, New York 1980.

[A2] W. Abikoff, Degenerating familes of Riemann surfaces, Annals of Math. 105 (1977), $29-44$.

[B] L. Bers, Spaces of degenerating Riemann surfaces. Ann. of Math. Studies 79, Princeton Univ. Press, Princeton, NY 1974, 43-55.

[Be] A. Beardon, On the Geometry of Discrete Groups, Graduate Texts in Mathematics 91, Springer-Verlag, New York 1983.

[Ch] I. Chavel, Eigenvalues in Riemannian Geometry, Academic Press, New York 1984.

[CC] B. Colbois and G. Courtois, Les valeurs propres inférieures à $1 / 4$ des surfaces de Riemann de petit rayon d'injectivitè, Comment. Math. Helvetici 64 (1989), 349-362. 
[DP] E. D'Hoker and D. Phong, On determinants of Laplacians on Riemann surfaces, Comm. Math. Phys. 105 (1986), 537-545.

[DJ1] J. Dodziuk and J. Jorgenson, On the spectral asymptotics and geometry of degenerating hyperbolic three manifolds, in: Extremal Riemann Surfaces, J. R. Quine and P. Sarnak (Eds.), AMS Contemp. Math. 201 (1996), 191-206.

[DJ2] J. Dodziuk and J. Jorgenson, Spectral asymptotics on degenerating hyperbolic 3-manifolds. Memoirs of AMS. To appear.

[He1] D. A. Hejhal, Regular b-groups, degenerating Riemann surfaces, and spectral theory, Volume 437 of Memoirs of the American Mathematical Society, American Mathematical Society, Providence, R.I. 1990.

[He2] D. A. Hejhal, The Selberg Trace Formula for PSL(2, R), vol. 1, Lecture Notes in Mathematics 548, Springer-Verlag, New York 1976.

[He3] D. A. Hejhal, The Selberg Trace Formula for PSL(2, R), vol. 2, Lecture Notes in Mathematics 1001, Springer-Verlag, New York 1983.

[HJL1] J. Huntley, J. Jorgenson and R. Lundelius, On the asymptotic behavior of counting functions associated to degenerating hyperbolic Riemann surfaces. J. Functional Analysis 149 (1997), 58-82.

[HJL2] J. Huntley, J. Jorgenson and R. Lundelius, A heat kernel approach to the Selberg zeta function for compact 2 and 3 manifolds. In preparation.

[JLa1] J. Jorgenson and S. Lang, Artin formalism and heat kernels. J. Reine Angew. Math. 447 (1994), 165-200.

[JLa2] J. Jorgenson and S. Lang, Explicit formulas for regularized products and series, in: Explict Formulas, Lecture Notes in Mathematics 1593, Springer-Verlag, New York 1983, 1-34.

[JLu1] J. Jorgenson and R. Lundelius, Convergence of the heat kernel and the resolvent kernel on degenerating hyperbolic Riemann surfaces of finite volume, Quaestiones Mathematicae 18 (1995), 345-363.

[JLu2] J. Jorgenson and R. Lundelius, Convergence theorems for relative spectral functions on hyperbolic Riemann surfaces of finite volume, Duke Math. J. 80 (1995), 785-819.

[JLu3] J. Jorgenson and R. Lundelius, Convergence of the normalized spectral function on degenerating hyperbolic Riemann surfaces of finite volume. J. Functional Analysis 149 (1997), 25-57.

[Lu] R. Lundelius, Asymptotics of the Determinant of the Laplacian on hyperbolic surfaces of finite volume, Duke Math. J. 71 (1993), 212-242.

[M] H. P. McKean, Selberg's trace formula as applied to a compact Riemann surface, Comm. Pure and Appl. Math. 25, (1972) 225-246.

[MP] S. Minakshisundaram, and A. Pleijel, Some properties of eigenfunctions of the Laplace operator on Riemannian manifolds, Can. Jour. Math. 1 (1949), 242-256.

[Mü] W. Müller, Spectral theory for Riemannian manifolds with cusps and a related trace formula, Math. Nachr. 111 (1983), 197-288.

[R] B. Randol, Cylinders in Riemann surfaces, Comm. Math. Helv., 54 (1979), 1-5.

[Sa] P. Sarnak, Determinants of Laplacians, Comm. Math. Phys. 110 (1987), 113-120.

[Se] A. Selberg, Harmonic Analysis and discontinuous groups in weakly symmetric Riemannian spaces with applications to Dirichlet series, J. Indian Math. Soc. B. 20 (1956), $47-87$.

[Wo] S. A. Wolpert, Asymptotics of the spectrum and the Selberg zeta function on the space of Riemann surfaces, Comm. Math. Phys. 112 (1987), 283-315.

Jay Jorgenson

School of Mathematics

Institute for Advanced Study

Princeton, NJ 08540

USA

(Received: January 27, 1997)
Rolf Lundelius

Department of Mathematics

University of the Witwatersrand

P.O. Wits 2050

South Africa 